\title{
Diseño de una propuesta de formación binacional en competencias digitales con docentes de educación superior en tiempo de Covid-19
}

Design of a Proposal for Binational Training in Digital Skills with Higher Education Teachers in Time of Covid-19

\author{
Wilder Banoy Suárez \\ Corporación Universitaria minuto de Dios (Uniminuto), Colombia \\ profe.wilder@gmail.com \\ ORCID 0000-0002-5807-2715
}

\section{RESUMEN}

El presente artículo tiene como objetivo compartir las fases de diseño, ejecución y análisis de resultados de un proceso de formación vinculado al desarrollo de competencias digitales, impartido desde Colombia y dirigido a docentes de una institución de educación superior en México; lo anterior, en el marco de situaciones emergentes producto de la Covid-19 y desde la perspectiva de la Clase invertida o Flippedclassroom. La necesidad de mejorar sustancialmente el nivel de esas competencias en la población se hizo evidente en tiempos de pandemia, concretamente en cuatro aspectos, planificación y ejecución de enseñanza híbrida con TIC, selección y creación de objetos virtuales de aprendizaje, diseño y desarrollo de entornos digitales de enseñanza y posibilidades de tutoría y evaluación en línea. El diseño curricular fue estructurado y adaptado a las necesidades particulares de formación, para un ciclo propedéutico en modalidad de diplomado entre julio y diciembre de 2020. El proceso finalizó con el listado para certificación de $82.9 \%$ de los docentes aprendices y aprobación por parte de los directivos de la universidad para la segunda cohorte en el segundo semestre de 2021.

Palabras clave: educación superior, formación de docentes, competencia digital, Covid-19, clase invertida

\section{ABSTRACT}

The objective of this article is to share the phases of design, execution, and analysis of results of a training process linked to the development of digital competencies taught from Colombia and aimed at teachers of a higher education institution in Mexico. This aim is framed in the emerging situations resulting from Covid-19 and from the perspective of the inverted class or flipped classroom. The need to substantially improve the level of these skills in the population became evident in times of pandemic, specifically in four aspects, planning and implementation of hybrid teaching with ICT, selection and creation of virtual learning objects, design and development of digital teaching environments, and online tutoring and assessment possibilities. The curriculum design was structured and adapted to the particular training needs for an induction cycle in the form of a diploma between July and December 2020. The process ended with the listing for certification of $82.9 \%$ of the apprentice teachers and the university managers' approval for the second cohort in the second semester of 2021.

Keywords: higher education, teacher training, digital competence, Covid-19, flipped classroom 


\section{INTRODUCCIÓN}

En palabras de De Zubiría (2006, p. 61), “evaluar es formular juicios de valor acerca de un fenómeno conocido, el cual vamos a comparar con unos criterios que hemos establecido de acuerdo a unos fines que nos hemos trazado"; esta acción se hace a cada instante, en cualquier lugar. De ahí que la educación, comprendida como un fenómeno aparentemente conocido, esté siendo evaluada por los estudiantes como nunca antes, con sus actitudes, niveles de motivación, compromiso, interés, y acompañamiento de sus familias en procesos formativos. En esa evaluación, tal como lo afirma Banoy (2020, p. 22):

Los aprendices demandan cambios, el mundo que les rodea se ha transformado radicalmente, pero el sistema educativo sigue prácticamente igual al existente a comienzos del siglo XIX. Las dinámicas de la inmediatez de la información y comunicación, y su multidireccionalidad no sólo en medios físicos sino digitales, permiten inferir que la educación debe ir a la par de esos cambios; claro está, sin desconocer los aportes de las teorías y modelos pedagógicos precedentes.

La influencia de los efectos colaterales de la Covid-19 aceleró dinámicas de desarrollo de competencias digitales en todos los campos de acción en los que el ser humano basa su comunicación en el contacto directo, incluyendo, por supuesto, los diversos y dispares escenarios educativos con sus respectivas comunidades en todo el orbe. Esas competencias han sido clasificadas por Cabero y Martínez (2019, p. 257) como "generales -cognitivas, sociales, comunicativas, tecnológicas y personales- y específicas -de planificación y organización del curso, didácticas y de evaluación, de manejo del ambiente en la sala de clase y de reflexión pedagógica e investigación-acción”.

En este escenario inesperado, las mencionadas competencias digitales fueron evaluadas no por medios tradicionales como exámenes o reportes, sino por las mismas situaciones emergentes que surgieron en la complejidad desencadenada por la pandemia; una complejidad que involucró todos los escenarios ligados a la educación y, por supuesto, como lo sustenta Rodríguez (2017, p. 425), a la forma- 
ción docente "a partir de la tríada currículum-educación-cultura", el currículo desde sus componentes fundamentales, educación sobre el impacto de la práctica pedagógica y la cultura desde la perspectiva de seres que forman parte de un contexto local y planetario. Esa complejidad, en palabras de Morin (2011, p, 32), es efectivamente "el tejido de eventos, acciones, interacciones, retroacciones, determinaciones, azares, que constituyen nuestro mundo fenoménico". Ahora más que nunca, se hacen notorios los tres aspectos que según Cabero y Aguaded (2013, p. 15) forman parte de esa complejidad:

En primer lugar, que las aulas han cambiado considerablemente y que la presencia de las tecnologías en ellas es cada vez más usual; en segunda instancia, que las características sociocognitivas de los estudiantes actuales son muy diferentes -sin tener que ser etiquetados como mejores o peores-, a las de los estudiantes de comienzo del siglo XX; entre otros motivos, porque las tecnologías no sólo nos ofrecen información, sino también reorganizan y modifican nuestra estructura cognitiva y formas de procesamiento, y ellos, al interaccionar con la red, los multimedia y los videojuegos, presentan estilos de procesamiento diferentes; y por último, nos tendríamos que interpelar en qué aula en concreto -en la presencial o la virtual- desarrollaríamos la enseñanza.

En este escrito se exponen, precisamente, esos tres aspectos y cómo se convirtieron en soporte y eje medular para la elaboración de un marco teórico y el diseño de un trabajo colaborativo entre el autor de la propuesta de formación -desde Colombia- y una institución de educación superior en México - Instituto Yucatán Península Misión o IYPM-. En otras palabras, se comparte con la comunidad académica un proceso telecolaborativo de formación para el desarrollo de competencias digitales docentes (CDD) en tiempos de Covid-19. Lo anterior, en el marco de la Clase Invertida, Aula Inversa o Flipped Classroom; comprendida por muchos como un modelo pedagógico, metodología, metaestrategia, en la que:

se transfiere la instrucción directa del espacio grupal al individual. Para ello el contenido básico es estudiado en casa con material aportado por el profesor y el aula se convierte en un espacio de 
aprendizaje dinámico e interactivo, donde el maestro guía a los alumnos mientras éstos aplican lo que aprenden y se involucran en el objeto de estudio de forma creativa (Santiago y Bergmann, 2018, p. 24).

De ahí que la presente publicación surja, en primer lugar, por la necesidad de entender la escuela más que como un lugar físico, como una oportunidad de aprender de forma ubicua y atemporal por medio de la tecnología y los avances investigativos propios de este milenio. En esa dirección, Robinson y Aronica (2015, p. 19) resumen el concepto de escuela como "una comunidad de personas que se reúnen para aprender juntas. Escuela en su acepción del término, comprende la educación en casa, la no escolarización y los encuentros informales tanto en persona como en línea desde la guardería hasta finalizada la universidad”.

Es claro, entonces, que sin desconocer los logros de los modelos pedagógicos heteroestructurantes o magistrocentristas en los que el maestro es centro del proceso -como sucede en el conductismo- o autoestructurantes, en los que el estudiante es el pilar fundamental, como la escuela activa o el constructivismo (De Zubiría, 2015); se requieren nuevas posibilidades de enseñanza y aprendizaje con una infinidad de matices que se adapten a la realidad global, continental, nacional y regional en cada comunidad. Al respecto, Banoy (2020, p. 23) sostiene que "se ha emitido esta misiva en incontables discursos, planes políticos, conferencias, simposios y congresos, pero son pocos los documentos o experiencias que de forma concreta ofrecen alternativas para este tipo de transformaciones".

Cada modelo pedagógico ha aportado valiosos conceptos a las prácticas de docentes en los dispares rincones del globo. La idea, entonces, no es desvirtuar esos aportes; por el contrario, sustentar en los mismos la necesidad de postular nuevos diseños pedagógicos para los distintos espacios y momentos de enseńanza y aprendizaje, adaptándolos a la complejidad del mundo, y que además se basen en una inclinación natural por la creatividad, el razonamiento crítico y el aprendizaje con significado, todo para que, en consecuencia, los estudiantes puedan enfrentarse así a la incertidumbre y situaciones emergentes impredecibles. 
Desde la investigación y la práctica pedagógica deben surgir propuestas que propendan por acciones concretas en las distintas formas de vivir el aprendizaje; la educación tiene que mutar de miles de formas para adaptarse al mundo actual y sus particularidades, tal como ha sucedido con las transformaciones educativas derivadas de la pandemia global de 2020 y 2021 -en el momento en que se escribe este artículo se presenta el tercer pico de pandemia-. A su manera, Morin y Delgado (2017, p. 8) se ligan con esta idea al afirmar que "la alternativa al abismo es la metamorfosis, pero ésta no llegará sin acciones".

Ese tipo de acciones disruptivas surgieron también en la educación superior, al igual que los distintos ciclos educativos que se vieron afectados por los efectos colaterales de la Covid-19; si bien ya era una necesidad para los actores que formaban parte del proceso pedagógico identificar posibles maneras de evolucionar sus prácticas educativas con base en los avances tecnológicos propios de la tercera década del siglo XXI, esta situación particular expuso la necesidad de adquirir competencias digitales que se ajustaran a la realidad educativa global y local en pandemia.

La crisis develó falencias sustanciales en las competencias digitales tanto de docentes, como de padres de familia y estudiantes. Esta publicación hace referencia puntual a las primeras; se comparte con la comunidad académica una experiencia de formación diseñada, ejecutada y finalizada en 2020 .

La necesidad de formación surgió en abril, luego del comienzo de la virtualidad total por la pandemia; era sustancial acelerar procesos de desarrollo de las mencionadas competencias y se consideró imperativo por parte de las directivas de la universidad buscar posibilidades que permitieran, en primer lugar, la actualización en el campo en mención y, en segunda instancia, migrar de la clase tradicional la metodología Aula Inversa, Clase Invertida o Flipped Classroom en sus estrategias pedagógicas de pregrado y posgrado, para una futura alternancia con modelos educativos híbridos e innovadores en el IYPM, metas claras que se convirtieron en el derrotero de la propuesta de formación docente.

Sobre el contexto curricular de la institución, vale la pena aclarar que la oferta académica en pregrado se enfoca en ocho progra- 
mas: administración de empresas, derecho, pedagogía, ingeniería en sistemas computacionales, teología, marketing y comercialización, contaduría y trabajo social. Mientras que, en posgrado, la oferta es de dos maestrías: tecnología educativa y gestión escolar. Todos los programas pueden cursarse en modalidad presencial (MP), con carga horaria establecida, y clases sincrónicas; modalidad ejecutiva (ME) con presencialidad de un día en fin de semana y tutorías virtuales en los días restantes por medio del Ambiente Virtual de Aprendizaje Google Classroom. Finalmente, la modalidad virtual, centrada en no presencialidad y uso de la misma plataforma. Los docentes que cursaron la propuesta de formación compartida en este artículo, orientan clases tanto en los programas académicos mencionados como en las tres modalidades.

En síntesis, el objetivo principal se enfocó en diseñar una propuesta pedagógica para desarrollar competencias digitales docentes (CDD) en profesores de educación superior, desde la perspectiva de clase invertida, concretamente en: planificación y ejecución de enseñanza híbrida con TIC, selección y creación de objetos virtuales de aprendizaje (OVA), diseño y desarrollo de entornos digitales de enseñanza y posibilidades de tutoría y evaluación en línea (Area-Moreira, 2019).

Así pues, la clase invertida o Flipped Classroom es una estrategia pedagógica -modelo para algunos- en la que, según el Centro de Innovación en Tecnología y Educación de la Universidad de los Andes en Colombia, Conecta-TE (2013, s. p.):

se aprovechan las oportunidades que brindan las Tecnologías de Información y Comunicación (TIC) para invertir la clase magistral tradicional y ofrecer a los estudiantes otras formas preparadas por cada profesor para aproximarse al conocimiento antes de la clase presencial -video de clase, podcasts, presentaciones interactivas, lecturas enriquecidas y otras-. De esta manera, los estudiantes preparan previamente la lección, de una manera totalmente autónoma e independiente en tiempos extra aula atendiendo a sus propios ritmos e intereses. Así, durante la clase, el tiempo se dedica a la realización de actividades basadas en la interacción con el conocimiento, con sus pares y con el profesor para solución de inquietudes, orientación y realimentación constante. 
Luego del contacto académico entre las partes y de comprender la necesidad particular de aprendizaje con respecto al desarrollo de competencias digitales, en julio de 2020 se elaboró el diseño curricular de la propuesta pedagógica en formato de diplomado, llamado Clase Invertida, nuevas tendencias en educación mediadas por tecnología. El trabajo fue estructurado con los directivos del IYPM, administrativos y personal técnico para adaptarlo a la realidad y contexto de la institución educativa. En agosto se hicieron los ajustes respectivos y en septiembre comenzó formalmente el proceso de formación, que finalizó en la tercera semana de diciembre; los detalles en secciones venideras.

La plataforma Google Classroom en su versión premium fue el LMS elegido para administrar recursos, sistema de evaluación, formatos documentales propios de la universidad, y todos los elementos que formaron parte del ecosistema virtual del aprendizaje. Aquí es de alta relevancia recordar que las plataformas LMS, según Torras (2018, p. 3),

son espacios virtuales de aprendizaje orientados a facilitar la experiencia de capacitación a distancia, tanto para instituciones educativas como para empresas. LMS es el acrónimo en inglés de Learning Management System, que podría traducirse como sistemas para la gestión de aprendizaje. Este sistema permite la creación de "aulas virtuales" donde se produce la interacción entre tutores y alumnos. También se pueden hacer evaluaciones, intercambiar archivos y participar en foros y chats, además, de otras muchas herramientas adicionales.

Esta plataforma fue elegida por dos razones principales. En primer lugar, porque era el LMS que la universidad empleaba en versión de pago; en otras palabras, se contaba con todos los servicios de la suite de Google, incluyendo, por supuesto, la grabación de clases, aspecto muy importante para los estudiantes que por alguna dificultad no podían acceder a los encuentros sincrónicos. En segundo lugar, por su practicidad y versatilidad, tanto en la administración del aula como en el uso e interacción en la misma, sea desde equipos de escritorio o dispositivos móviles. 
Gran parte de la importancia de este ejercicio académico radicó en su característica de originalidad en el contexto latinoamericano; en primera instancia, por el tipo de contenido abordado, su origen por emergencia global, la colaboración entre los dos países, y en cuarto lugar, por la adaptación cultural entre el autor de la propuesta de formación, directivos, personal técnico-administrativo y, por supuesto, los docentes aprendices con necesidades específicas de enseñanza y aprendizaje.

Aquí radica la necesidad de compartir por medio de este artículo la experiencia, con el fin de que se convierta en referente continental para instituciones de educación superior que busquen rediseñar estrategias de enseñanza y aprendizaje por medio del desarrollo de competencias digitales en sus docentes y cuyo eje teórico-práctico sea la innovación educativa por medio de la Clase Invertida o Aula Inversa.

En el segundo apartado de esta publicación se exponen los seis elementos del diseño curricular que sustentaron la propuesta; así pues, se describen los contenidos, la secuencia, la metodología, los recursos, la evaluación y, por supuesto, los propósitos de aprendizaje y competencias esperadas, estructura del diseño curricular propuesto por De Zubiría (2013).

En el acápite subsiguiente, se describen los resultados y logros obtenidos en términos de competencias digitales desarrolladas, asistencia, cumplimiento de actividades - descripción particular y general-, resultados de evaluación y porcentajes de aprobación para posterior certificación. Todo lo anterior como preámbulo a la discusión y conclusiones, que tienen que ver directamente con los objetivos planteados en el mes de junio en la fase de diseño y planeación, y la experiencia durante los meses de ejecución y resultados obtenidos.

Fue una experiencia entre actores educativos de dos países hermanos que, en tiempos de pandemia y virtualidad, transformaron la adversidad en una oportunidad para fortalecer competencias digitales en los docentes de educación superior por medio del teletrabajo, la telepresencia y los medios digitales. Esta sinergia impulsó un impacto real en toda la comunidad educativa, ya que desde el primer cuatrimestre de 2021 se implementa la clase invertida en la universidad; además, se espera que la segunda cohorte del diplomado forme 
alrededor de 45 docentes y administrativos de la misma comunidad en el segundo semestre de 2021.

Para cerrar esta introducción, es menester citar a Santiago y Bergmann (2018, p. 22), con sus recomendaciones para cambiar las clases tradicionales y adaptarlas a los estudiantes al aprendizaje activo, los principios básicos que plantean son:

Apoyar la personalización, el aprendizaje individual a gran escala. Preparar a los estudiantes para un futuro desconocido versus un pasado que desaparece a toda velocidad. Ser flexibles ante grandes cambios, que además se producen de forma acelerada. Permitir seguir usando lo que de verdad funciona en educación y eliminar aquello que ya no sirve. Basarse en el aprendizaje activo, que involucra y hace dueños de su propio aprendizaje a los estudiantes. Ayudar a desarrollar profesores y estudiantes de calidad, comprometidos con el aprendizaje y el bienestar general.

Es muy difícil aplicar al pie de la letra y en realidades particulares metodologías que han tenido éxito en algún lugar del orbe; cada comunidad y grupo de estudiantes tiene características singulares; el reto como docentes, directivos y dirigentes es organizar procesos eficientes en los que se identifiquen las verdaderas necesidades educativas del alumnado, teniendo como referencia las dinámicas globales y cambios acelerados en los que las comunidades están inmersas. Sin más preámbulo, se explica a continuación la propuesta de formación.

\section{METODOLOGÍA}

En la fase previa al diseńo curricular, cuatro encuentros durante el mes de junio con personal directivo y administrativo del IYPM permitieron identificar, desde Colombia, el sistema organizacional interno de comunicación, plataforma virtual de formación, dinámicas institucionales y competencias por desarrollar. Esos encuentros cimentaron la elaboración de la propuesta.

En otras palabras, las variables que se vincularon a la fase de planeación, diseño y ejecución, se ligaron de forma directa a la realidad 
de la institución educativa por medio de evaluación diagnóstica, que normalmente "se da al inicio del proceso de enseñanza y de aprendizaje, y permite obtener un juicio de valor y una toma de decisiones pedagógicas para fundamentar el planteamiento didáctico en concordancia con los aprendizajes previos de los estudiantes" (Ruz, 2018, p. 21).

La evaluación diagnóstica se sustentó en dos líneas de acción. Aplicación de dos instrumentos en formato cuestionario; el primero, sobre caracterización de la población e ideas fuerza, para identificar el tipo de percepción frente a algunos conceptos y niveles de conocimiento, habilidades y destrezas con respecto a los mismos -dos secciones con 30 preguntas en total-. El segundo cuestionario, sobre competencia digital docente -aplicado también como prueba de salida al finalizar el diplomado-, tuvo cuatro secciones, cada una ligada a uno de los aspectos descritos en el resumen e introducción del artículo -60 preguntas en escala tipo Likert-. La segunda línea de acción del diagnóstico fue el diálogo no estructurado con directivos y personal administrativo de la universidad, durante los meses de planificación y diseño de la propuesta. Lo anterior, con el fin de identificar necesidades particulares de aprendizaje y competencias previas al diplomado.

Esta evaluación fue generada en conjunto con personal directivo $y$ administrativo en los meses de junio y julio, para identificar necesidades emergentes de aprendizaje en términos de competencias digitales de docentes, directivos y administrativos en cuatro funciones específicas: planificar y gestionar situaciones de enseñanza presencial con TIC; seleccionar y crear objetos virtuales de aprendizaje; diseñar y desarrollar entornos digitales de enseñanza, y tutorizar y realziar evaluación continua en línea (véase figura 1).

Los aspectos que se describen a continuación, se ajustan a los elementos que componen la propuesta curricular, claro está, rescatando dos propiedades del currículo; la primera, que es un concepto polisémico y complejo de definir, y en segundo lugar, que para esta investigación se tuvo en cuenta la perspectiva de Zabalza (2007, p. 21 ), que lo contempla como "un proyecto formativo que se pretende llevar a cabo en una institución formativa”; un proyecto dinámico, cambiante y adaptado al contexto real de la práctica pedagógica. 
Figura 1. Funciones de docentes competentes en entornos virtuales

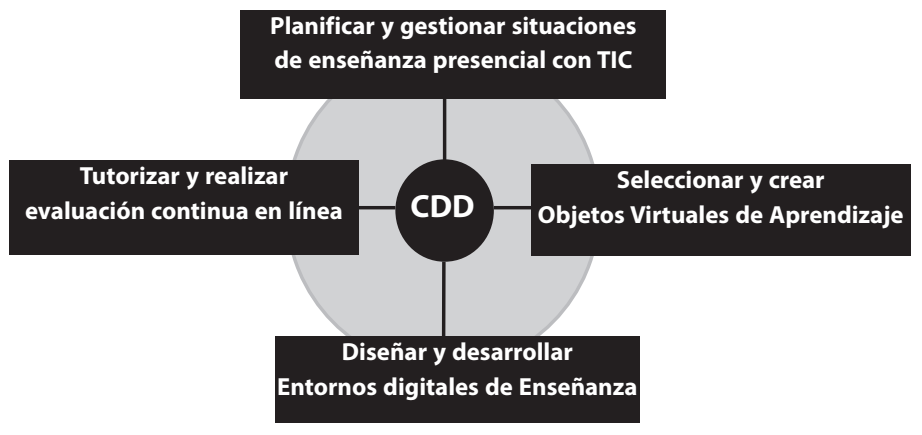

Fuente: elaboración propia con base en Area-Moreira, 2019.

Como se expuso en el apartado introductorio, se eligió el diseño curricular de De Zubiría (2013) por su concreción y facilidad de adaptación; seis elementos constitutivos -contenidos, secuencia, metodología, recursos, evaluación y propósitos de aprendizaje-, que estructuraron las tres fases de la propuesta de formación -diseño, ejecución y análisis-síntesis (véase figura 2).

- Figura 2. Diagrama de Gantt, tres fases de propuesta de formación

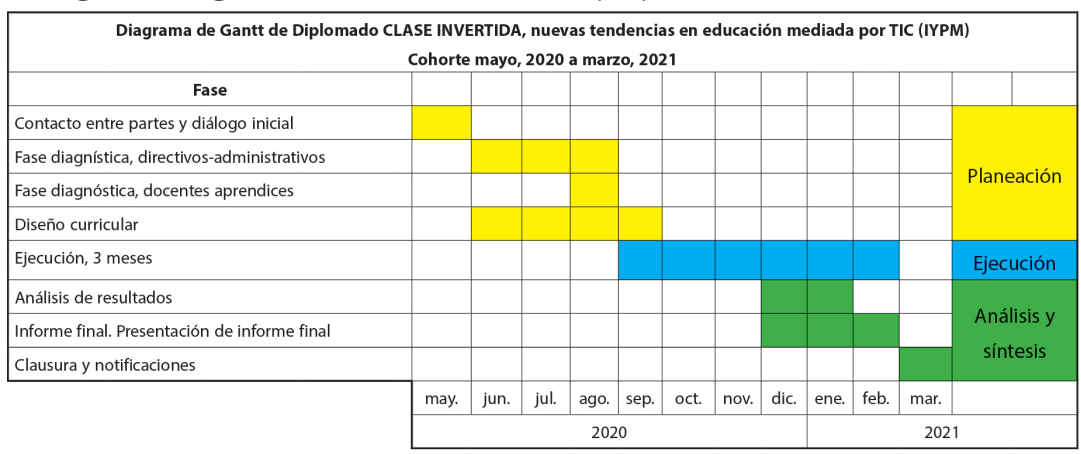

Fuente: elaboración propia.

Tal como se evidencia, el contacto entre las partes, planeación, diagnóstico y diseño curricular se hizo entre mayo y septiembre, la ejecución o aplicación se llevó a cabo desde septiembre hasta la tercera semana de diciembre 2021 y la fase final culminó en marzo de 2021. 
El primer elemento curricular se ajustó a los objetivos y competencia de aprendizaje por desarrollar en docentes, directivos y administrativos aprendices del IYPM. En esa dirección, la competencia fue comprendida como "una interacción de saberes" (Ríos y Herrera, 2017, p. 1077), saberes que se vincularon a lo conceptual, actitudinal y procedimental. Por todo lo anterior y luego del diagnóstico inicial, la competencia general se concretó como se refleja en el cuadro 1.

Cuadro 1. Competencia y elementos de competencia del diplomado Clase Invertida

\begin{tabular}{|l|l|}
\hline Competencia general & $\begin{array}{l}\text { Incorpora la Clase invertida en su práctica pedagógica con el fin de propiciar } \\
\text { cambios sustanciales en la forma de enseñar y aprender en el contexto } \\
\text { académico del IYPM. }\end{array}$ \\
\hline $\begin{array}{l}\text { Elemento de } \\
\text { competencia 1: saber }\end{array}$ & $\begin{array}{l}\text { Conocer variables relacionadas con la metodología Clase invertida y tendencias } \\
\text { pedagógicas mediadas por tecnología que pueden vincularse a la misma. }\end{array}$ \\
\hline $\begin{array}{l}\text { Elemento de } \\
\text { competencia 2: ser }\end{array}$ & $\begin{array}{l}\text { Apropiar la Clase invertida como nuevo medio para mejorar los niveles de } \\
\text { motivación en las actividades de enseñanza y aprendizaje en el contexto } \\
\text { académico del IYPM. }\end{array}$ \\
\hline $\begin{array}{l}\text { Elemento de } \\
\text { competencia 3: hacer }\end{array}$ & $\begin{array}{l}\text { Diseñar estrategias pedagógicas reales con base en la metodología Clase } \\
\text { Invertida que presenten alternativas de solución a dificultades de enseñanza y } \\
\text { aprendizaje en la comunidad educativa del IYPM. }\end{array}$ \\
\hline
\end{tabular}

Fuente: elaboración propia.

Los tres elementos de competencia (EC) se adaptaron a las cuatro funciones de docentes competentes en entornos virtuales de aprendizaje (figura 1) y a las necesidades educativas del público objetivo. De tal manera que el EC1 se ligó a los niveles cognitivos que desde la perspectiva de Bloom se ajustan al orden inferior -comprender y recordar-, para ir escalando progresivamente al orden superior (Santiago, Díez y Andía, 2017). Dicho de otro modo, el EC1 se vinculó de forma directa a la explicación de conceptos, variables y tendencias que forman parte de la Clase invertida; mientras que el EC2, se enfocó en lo actitudinal y axiológico. Finalmente, el EC3 se ajustó a las semanas finales, donde un taller práctico y el diseño de un proyecto final de enseñanza aprendizaje gestada en grupo por los docentes aprendices, se convirtieron en requisitos para aprobar y culminar este ciclo propedéutico. 
Ahora bien, se expone, además, en este apartado, el segundo elemento curricular, los contenidos. El diplomado se basó en ocho unidades temáticas. La primera -conceptos generales- fue diseñada como preludio a la unidad que explica la Clase Invertida; fue necesario abordar principios y términos pedagógicos como la importancia de la didáctica, su relación con los conceptos de lúdica, diversión, motivación, juegos y modelos pedagógicos como el autoestructurante, heteroestructurante e interestructurante; este último, también conocido como dialogante, apunta a que se reconozca tanto el papel activo del estudiante en el aprendizaje, como el papel esencial y determinante de los mediadores en este proceso (De Zubiría, 2006).

En ese orden de ideas, para comprender los modelos pedagógicos junto a los actores y papeles que los componen, en la segunda parte de la primera unidad se generó una aproximación al concepto de currículo, sus seis aspectos constitutivos y el enfoque ligado al desarrollo de competencias. Al finalizar, entraron en escena términos bastante empleados en estos días de crisis mundial: innovación educativa mediada por tecnología, competencias digitales docentes (CDD), su clasificación y componentes.

En otras palabras, la unidad uno se convirtió en una plataforma base a nivel conceptual y metacognitivo, con el fin cimentar en las siete unidades restantes diálogos pedagógicos reflexivos por parte de los diferentes actores que formaron parte del diplomado. El organizador gráfico se muestra en la figura 3.

Por su parte, la unidad dos tuvo como fin, en primera instancia, dar forma al concepto de Clase invertida, Clase inversa, Aula invertida, Aula inversa o Flipped Classroom, metodología con nombres diversos que apuntan al mismo sistema; exponer aspectos generales de su evolución y, por supuesto, analizar investigaciones, publicaciones, ideas, aportes y experiencia de los expertos Jonathan Bergmann, Aaron Sams, Raúl Santiago, Eric Mazur y Salman Khan (Banoy, 2020).

Para culminar, se abordó el término The flipped mastery o aprendizaje para el dominio, en el que según García-Barrera (2013, p. 5) "cada alumno puede seguir su propio ritmo de aprendizaje, ya que éste no se impone desde la clase en modo alguno"; concepto muy importante en una de las ideas fuerza del diplomado: todos aprenden de maneras diferentes y con ritmos desiguales. La unidad 
culminó con los cuatro pilares del aprendizaje invertido: ambiente flexible, cultura de aprendizaje, contenido intencional y educadores profesionales (FNL, 2014); esto, para apropiar mucho mejor las ideas sobre Flipped Classroom (véase figura 4).

- Figura 3. Organizador gráfico de Unidad 1

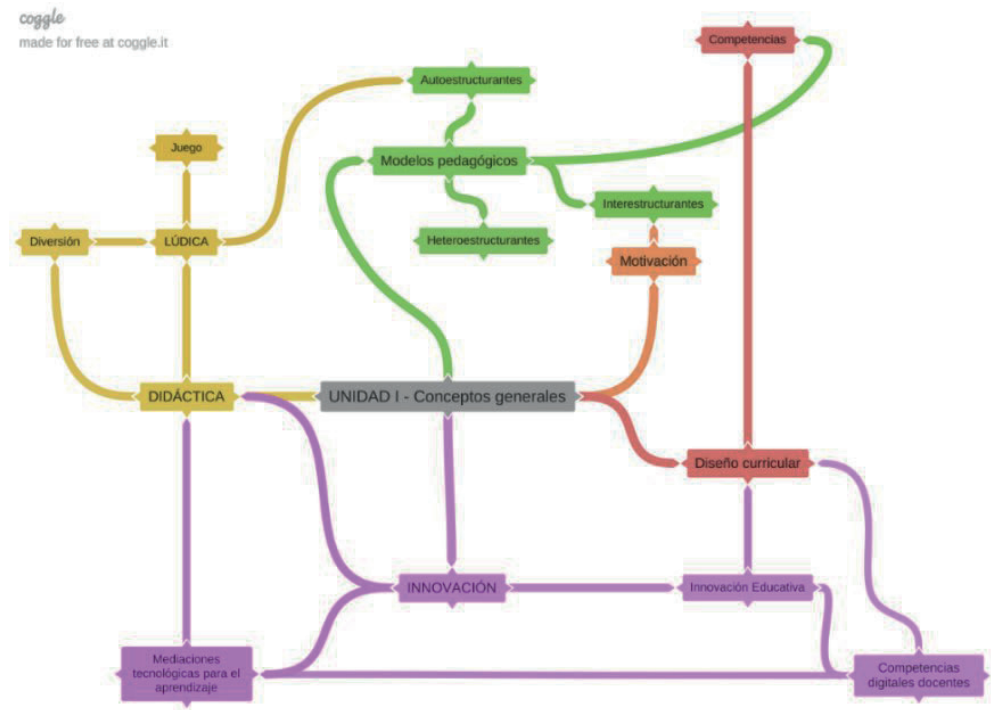

Fuente: elaboración propia.

- Figura 4 Organizador gráfico de Unidad 2

coggle

made for free at

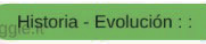

The flipped mastery

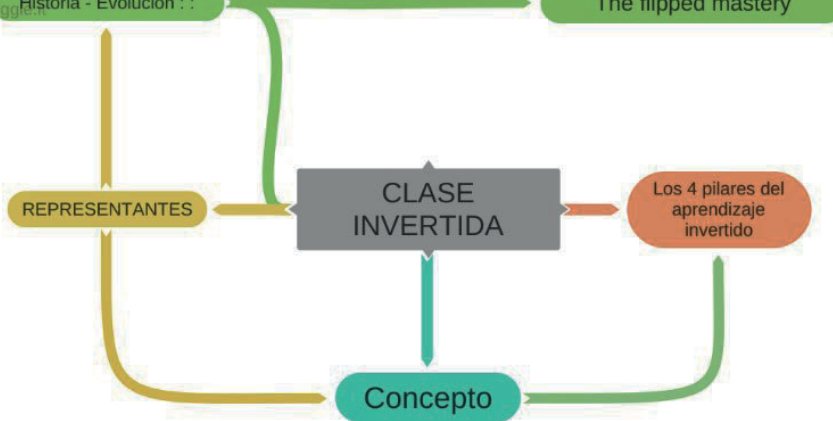

Fuente: elaboración propia. 
La unidad tres fue una sección sustancial; se ligó al papel del docente y del estudiante en la metodología clase invertida. Se resaltó que los docentes han de ser expertos en contenidos, sin que eso signifique que sean el centro del proceso educativo; deben conocer sobre tecnología, aprender a planificar, a diferenciar; son ahora entrenadores cognitivos más que fuentes del saber, aprender a diseñar preguntas -no es un proceso sencillo, así lo parezca- y, por supuesto, reconocerse como pilares fundamentales para motivar, de ahí la importancia de ser buenos conferenciantes (Santiago y Bergmann, 2018).

Luego, fue compartido el concepto de espacio individual y grupal y cómo cambian las actividades de alta y baja complejidad en esta estrategia. Precisamente, en la sección final, se ilustra el papel del estudiante en esta metodología y recomendaciones de las clases invertidas para el espacio individual. En ese orden de ideas, se gestó un diálogo sobre competencias digitales y los requerimientos en este campo para docentes, directivos, padres y, por supuesto, estudiantes (véase figura 5).

Figura 5. Organizador gráfico de Unidad 3

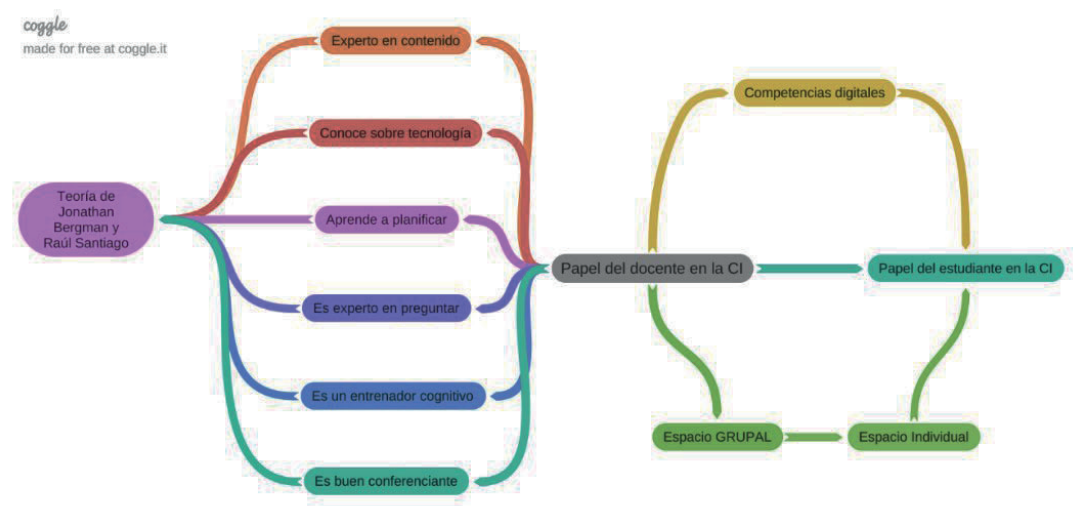

Fuente: elaboración propia.

En esa dirección, la unidad cuatro tuvo como eje las distintas formas de diseñar objetos virtuales de aprendizaje (OVA) interactivos, comprendidos como "aplicaciones de las TIC que median el proceso pedagógico” (Valero, Palacios y González, 2019), orientados al desempeño de estudiantes, principalmente en el espacio indi- 
vidual. Dicho de otro modo, en esta unidad finalizó la parte teórica formal (EC1), dando paso al ejercicio de metacognición (EC2) y por supuesto, la práctica (EC3).

Una idea medular se sustentó en que el desarrollo progresivo de herramientas online y aplicaciones, hace que día a día sean reevaluadas, en primer lugar, las metodologías que permiten la tecnología en términos pedagógicos y, en segunda instancia, las posibles estrategias que se basan en elementos que los estudiantes puedan tener cerca, para evitar un papel pasivo al abordar el material diseñado para el trabajo autónomo en el espacio individual; niveles recordar y comprender de Bloom (Blázquez y Tarazaga, 2017).

Se hizo énfasis en la experiencia al interactuar con el video -claro está, si es la opción seleccionada por el docente-, su utilidad, papel activo y motivacional, con técnicas como preguntas inmersas -videos enriquecidos-, hojas de guía o formatos online de preguntas y estrategias 3, 2, 1 en las que, según Santiago y Bergmann (2018, p. 75), los estudiantes "registran tres cosas que aprendieron del video, dos preguntas sobre su contenido y una duda persistente que aún tienen". Con esta última técnica, durante la clase, informan de los tres elementos que aprendieron, interactúan entre ellos sobre las dos preguntas y conversan con el maestro lo que no entendieron. Finalmente, se compartieron ejemplos para realizar evaluación sobre videos enriquecidos o lecturas con esa misma característica; aquí, el video de forma automática se pausa y se hacen preguntas en momentos específicos con distintos formatos, como tipo examen, encuesta, respuesta larga o llamados a la acción para interactuar con recursos externos (Banoy, 2021).

La anterior es una de las alternativas más atractivas para los estudiantes, porque pueden ir midiendo su progreso mientras que los docentes obtienen datos, lo que comúnmente se llama Analítica de Aprendizaje en tiempo real sobre: tiempo en pantalla destinado al video, número de intentos, cantidad de aciertos y errores, entre otros. Actualmente, los docentes pueden tener a disposición herramientas que cumplen con estas funciones, como EdPuzzle, PlayPosit, Vizia y otras. Para dar claridad sobre el concepto, Corona et al. (2019, p. 32), concluyen que la analítica de aprendizaje: 
puede hacer uso de cualquier herramienta o campo que le permita obtener, describir, analizar e interpretar datos sobre los alumnos y sus procesos de aprendizaje; de esta manera, las analíticas del aprendizaje permiten crear conocimiento que contribuya a mejorar el proceso de aprendizaje y su entorno.

Como complemento, se compartió con los docentes plataformas que además de permitir crear y alojar videos para marketing, educación, gestión empresarial y otros campos, también ofrecen la posibilidad de generar interacciones avanzadas; algunas con servicio completo gratuito (free), otras con servicios híbridos (freemium) y muchas con servicios exclusivos de pago (premium).

En esta unidad, se hizo especial hincapié -de manera simultánea- en las dificultades económicas que tienen las instituciones educativas, y en la opción de sustentar la necesidad pedagógica de uso de herramientas flipped frente a colegas y directivos, desde la perspectiva de un cambio de paradigma educativo, no como moda, sino como necesidad. Lo anterior con el fin de que sea asignado presupuesto para licencias o planes de suscripción que apunten a mejorar los resultados de las clases invertidas por medio del uso de herramientas específicas.

Con respecto a lo expuesto, se hizo énfasis en que cada docente determina en la metodología fipped la mejor manera de generar interactividad que se ajuste a sus clases, estudiantes y características del entorno, y busca la mejor combinación que permita esa interactividad, sea con herramientas como éstas, o simplemente con hojas de preguntas formuladas de forma previa al análisis del video por parte de los estudiantes en el espacio individual.

Por último, se reconocieron otras posibilidades para la elaboración de material pedagógico abordado por el estudiante en el espacio individual -distintas del video-; entre otras, los docentes, directivos y administrativos del IYPM identificaron plataformas para creación y edición de podcasts, simuladores, animaciones, realidad aumenta$\mathrm{da}$, presentaciones enriquecidas, textos planos y web interactivos o enriquecidos, entre otras (véase figura 6). 
שigura 6. Organizador gráfico de Unidad 4

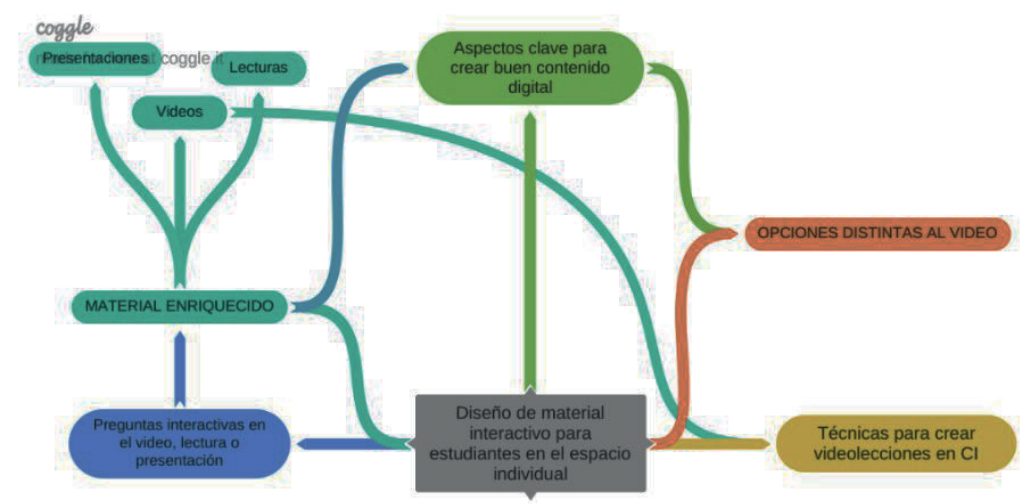

Fuente: elaboración propia.

Un punto de inflexión fue la unidad cinco y cómo aprovechar de forma eficiente el espacio grupal en Clase invertida, una pregunta que debe estar de manera constante en la mente de los educadores, para enlazar en su planeación de clases con los dos espacios utilizados en todas las sesiones que forman parte de la unidad de aprendizaje, bimestre, cuatrimestre, semestre o año académico completo. Aquí se hizo necesario recordar y ahondar en la pirámide y el diamante realista para la Clase invertida, expuesto en la parte inicial del curso; los niveles comprender y recordar se asocian al espacio individual, mientras que: aplicar, analizar, evaluar y crear con el espacio grupal. Esta sentencia fue una constante en las ocho unidades, con el fin de no perder el norte y propósito de aprendizaje en los docentes aprendices.

Si el material que el docente diseña para el espacio individual no es de fácil comprensión, terminará repitiendo la clase completa en el espacio grupal; de ser así, la estrategia pierde su esencia. Se aclaró, además, que dedicar 30 o 40 minutos a explicar los contenidos de una lectura, podcast, simulación o video de seis, ocho, diez o 15 minutos, haría que se desdibujara la idea de realizar una verdadera Clase invertida.

El objetivo es resolver dudas particulares, no todos los estudiantes comprenden los contenidos de la misma manera -punto sustancial-. Esas preguntas van a ser el termómetro que permitirá medir la 
calidad del material en línea compartido con anterioridad. Un error común es asumir que los estudiantes comprendieron los conceptos; aquí que el diálogo personal, intercambio de ideas y confianza generada para esas acciones son aspectos valiosos para identificar la evolución individual del aprendizaje.

De forma paralela, se pueden emplear estrategias metodológicas -algunas muy conocidas- en el espacio grupal, que propendan, en primer lugar, por consolidar lo explicado en el video o material flipped, aprovechando el tiempo que se dedicaba en la clase tradicional a la explicación magistral del profesor. En esta unidad, fueron revisadas algunas, como la enseñanza entre iguales, In Class Flip-recomendada cuando existan dificultades de equipos y conectividad-, aprendizaje basado en proyectos, juegos de roles y otras.

Para concluir esta unidad, se compartieron tendencias en educación mediadas por tecnología que se pueden asociar a la Clase invertida; entre otras, se generó un contexto académico sobre gamificación educativa, aprendizaje basado en juegos o ABJ, juegos serios, aprendizaje móvil (m-learning) y lineamientos de diseño de cursos en formato MOOC; el organizador gráfico de la unidad cinco concreta este planteamiento (véase figura 7).

- Figura 7. Organizador gráfico de Unidad 5

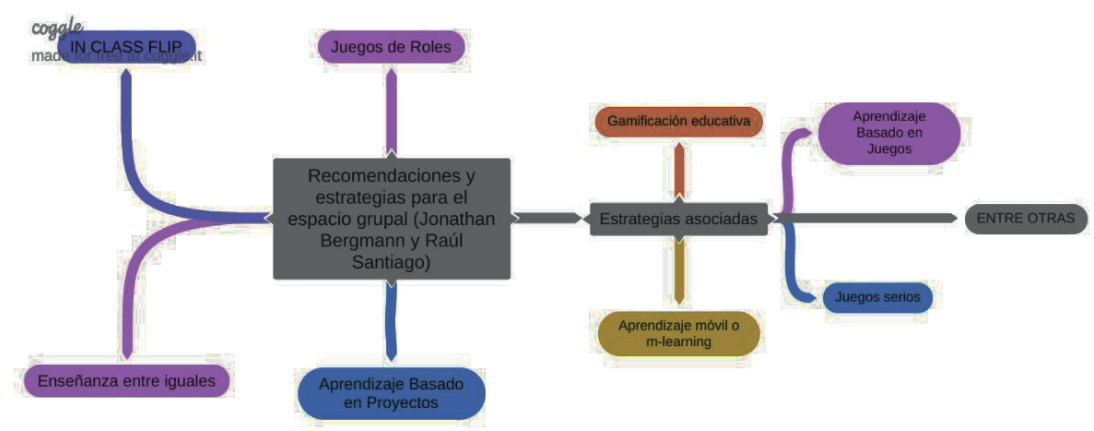

Fuente: elaboración propia.

Durante el tiempo crítico de pandemia en 2020, se hizo más notoria la gran brecha que en términos económicos y tecnológicos existe entre muchas comunidades educativas urbanas y rurales de Latinoamérica; al respecto, Orduz (2021) señala que, para el caso colombiano: 
Se sabe que el uso de móviles y el acceso a TV a escala nacional es amplio $-72 \%$ y $66 \%$ respectivamente-; sin embargo, el porcentaje de hogares con acceso a Internet es de 53\% y a computadores portátiles de $29 \%$. Las cifras son más inequitativas cuando se comparan las áreas rurales con las ciudades.

Situación que ha afectado no sólo a estudiantes y familias enteras, sino a los mismos docentes e instituciones donde laboran. Atendiendo al objetivo de la unidad seis, llamada Clase invertida en comunidades educativas con dificultades de acceso a tecnología, es claro que otra limitante para llevar a cabo esta metodología y que se vincula de forma directa al enunciado anterior, son las dispares condiciones entre la ruralidad y el sector urbano, o la falta de recursos en los dos escenarios. Factores como corrupción, intermitencia o ausencia de conectividad o del mismo servicio de energía eléctrica, y baja calidad de equipos informáticos forman parte las condiciones que varían según la particularidad de cada lugar.

Por tal razón, en esta corta unidad se presentaron posibilidades metodológicas para emplear Clase invertida en contextos con dificultades como las mencionadas; se resaltó que la creatividad de cada uno de los docentes se convierte en insumo fundamental para poder elaborar un diseño pedagógico propio y realista, claro está, con base en las necesidades específicas de la comunidad educativa. Se resaltó, además, que en el contexto educativo no existen recetas ni fórmulas mágicas; cada docente puede emplear una, dos, tres posibilidades o más y crear su propia mezcla para que el ejercicio de enseńanza aprendizaje sea motivador, desde la perspectiva de Clase Invertida, a pesar de las circunstancias.

Para culminar con este apartado, ligado al segundo elemento curricular de la propuesta de formación -los contenidos-, la unidad siete se enfocó en un taller práctico sobre la metodología, con herramientas, aplicaciones y recomendaciones sobre diseño, planeación, creación, edición, ubicación e integración curricular de objetos virtuales de aprendizaje (OVA) en formatos como: video educativo, imagen, infografías, animaciones, presentaciones, intros o animaciones de entrada para videos, materiales enriquecidos, audio (podcasts), aplicaciones para realimentación y evaluación tradicional 
e interactiva en tiempo real, juegos educativos virtuales, pizarras interactivas, comunicación y gestión de comunidades, muros colaborativos, videoconferencia y clases en línea, diseño gráfico educativo, gamificación y gestión de aula por medio de LMS.

Lo anterior sirvió como preludio a la última unidad del curso, en la que de los docentes aprendices finalizaron un proyecto pedagógico innovador, sostenible y realista, basado en Clase invertida. Los detalles más adelante, cuando se exponga el sistema de evaluación inmerso en la propuesta de formación binacional para el desarrollo de competencias digitales en la comunidad del IYPM.

Ahora bien, en el tercer elemento curricular -los recursos-, es pertinente destacar que los materiales pedagógicos fueron construidos siguiendo las directrices y formato educativo de la institución; esto con el fin de evitar traumatismos en la comunicación institucional. El LMS empleado como sitio virtual de referencia, y que sirvió para aprendizaje autónomo, comprendido este último como "una de las mejores herramientas del aprendizaje permanente" (Solórzano, 2017, p. 245), la socialización de material de apoyo y calificaciones, guías semanales, actividades de aprendizaje, entregas parciales y finales de productos académicos, ejercicios de autoevaluación y grabación de clases sincrónicas fue Google Classroom, "una herramienta TIC que, sumada a la capacidad y proactividad del docente, puede mejorar los procesos de enseñanza y aprendizaje favoreciendo que éstos sean ubicuos" (Kraus, Formichella y Alderete, 2019, p. 79).

En síntesis, los estudiantes pudieron acceder desde plataforma a presentaciones compartidas en clase, apuntes de unidad, constituidos por videos pregrabados con información relevante de las diferentes unidades, avisos semanales, lectura base -guía semanal-, diseñada con la intencionalidad de asegurar el acceso a fuentes de información y explicación de los temas del programa, y más de 180 enlaces para profundizar en conocimientos y metodologías alternativas de enseñanza; el documento tuvo una extensión final de 162 páginas, incluyendo referencias.

Además, los aprendices encontraron en plataforma actividades de aprendizaje, parciales y final -proyecto pedagógico-; lo anterior, desde el aprendizaje basado en proyectos, una metodología que permitió a los estudiantes convertirse en protagonistas de su propio 
aprendizaje y desarrollar su autonomía y responsabilidad, pues fueron los encargados de planificar, estructurar el trabajo y elaborar el producto para resolver la cuestión planteada (Banoy, 2020).

Para finalizar esta concisa descripción de los recursos empleados en la propuesta de formación, se destaca que, en el aula virtual, los aprendices realizaron una autoevaluación semanal, formada por preguntas clave; a través de los datos obtenidos se identificó el progreso tanto individual como grupal; de la misma forma, se diseñaron foros con la finalidad de propiciar interacción entre estudiantes semana a semana; se intercambiaron ideas constantemente y concretaron su conocimiento con la percepción de sus compañeros en cada unidad.

Es así como a través de los recursos, se evidenció un proceso de comunicación interactivo de construcción personal de significado, donde los alumnos organizaron, filtraron, codificaron, categorizaron y evaluaron la información y estructuraron su nuevo conocimiento.

En términos de secuencia curricular -cuarto elemento-, la presentación del diplomado fue en la tercera semana de septiembre en sesión sincrónica, citada desde Colombia por medio del LMS mencionado y con convocatoria oficial desde la plataforma de la universidad por parte de directivos y docentes. A partir de la fecha, doce semanas de estudio hasta diciembre, el ciclo culminó con sustentaciones finales de proyectos pedagógicos basados en Clase invertida. El material se compartió en plataforma cada lunes y las sesiones sincrónicas fueron los miércoles de 8:00 a 10:00 p.m., la sesión final fue de cuatro horas. La intensidad horaria puede verse en el cuadro 2.

- Cuadro 2. Intensidad horaria, diplomado Clase Invertida

\begin{tabular}{|l|c|l|}
\hline \multicolumn{1}{|c|}{ Ítem } & Cantidad de horas & \multicolumn{1}{c|}{ Fecha, 2020 } \\
\hline Presentación oficial (1) & 2 & Sábado 19 de septiembre \\
\hline Encuentros sincrónicos (12) & 24 & $\begin{array}{l}\text { Todos los miércoles de 8 } \\
\text { a 10 p.m. }\end{array}$ \\
\hline Trabajo autónomo en módulos I y II & 7 por semana (8 semanas) $=56$ & Primeras 8 semanas \\
\hline Trabajo autónomo en módulos III y IV & 9 por semana (4 semanas) = 36 & Últimas 4 semanas \\
\hline Socialización de propuestas finales & 4 & Miércoles 16 de diciembre \\
\hline Total de horas: & $2+24+56+36+4=122$ & \\
\hline
\end{tabular}

Fuente: elaboración propia. 
La clausura se hizo en marzo de 2021, luego de la revisión de procesos grupales e individuales, análisis de resultados y presentación de informe final a directivas de la Universidad.

Con respecto a la metodología-quinto elemento- el modelo pedagógico empleado tuvo un enfoque dialogante e interestructurante que, reconociendo el papel activo del estudiante en el aprendizaje, resaltó también el papel esencial de los mediadores en este proceso (De Zubiría, 2006). En el diplomado se presentó, entonces, un alto protagonismo del aprendiz con respecto a la construcción personal de conocimiento, con la orientación del docente tutor.

Este último -el docente tutor-, fue comprendido no como centro y eje del proceso, sino como guía. Los aprendices desarrollaron distintos niveles de aprehensión y desarrollo de competencias digitales y fue fundamental el alto nivel de motivación, dedicación, buenos hábitos de estudio en el espacio individual y disposición por aprender; el pensamiento crítico y aportes constructivos en el espacio grupal fueron sustanciales en la consecución de los objetivos.

Para ser consistente con la modalidad educativa y las condiciones del curso, la evaluación -sexto elemento curricular- formó parte del proceso mismo de aprendizaje, por lo que las actividades y tareas planteadas y realizadas por los participantes se consideraron elementos clave e importantes en el cumplimiento de los propósitos iniciales.

Tomando en cuenta, además, el enfoque metodológico de construcción del conocimiento empleado en el curso, se evaluó tanto la asistencia, como la participación en foros y actividades que reflejaron en su mayoría que dicho proceso de construcción se estuviera presentando.

Cada actividad de aprendizaje contó con rúbricas que detallaban indicaciones, categorías/aspectos a evaluar, procedimientos específicos y vínculo con el elemento de competencia al que se apuntaba. Lo anterior implicó, en consecuencia, alta relevancia de la participación en cada actividad para la aprobación del curso.

Como complemento al sistema descrito, se estableció un código de ética, responsabilidad, honestidad y comportamiento, entre todos los actores vinculados al ejercicio virtual de formación binacional para el desarrollo de CDD. 
A pesar de ser una propuesta de formación con contenidos y metodología que pueden mostrar resultados en distintos niveles educativos, se enfocó en la educación universitaria debido al alto nivel de autonomía y bajo nivel de prescripción de los estudiantes que forman parte de las tres modalidades de estudio en el IYPM, tanto en los programas de pregrado como los de posgrado.

\section{RESULTADOS}

El proceso de innovación pedagógica, según lo sustenta Santiago y Bergmann (2018), se basó en el planteamiento de preguntas específicas que originaron el diseño de la propuesta de formación. Las mismas, pueden verse en la figura 8 .

- Figura 8. Preguntas clave en el proceso de innovación

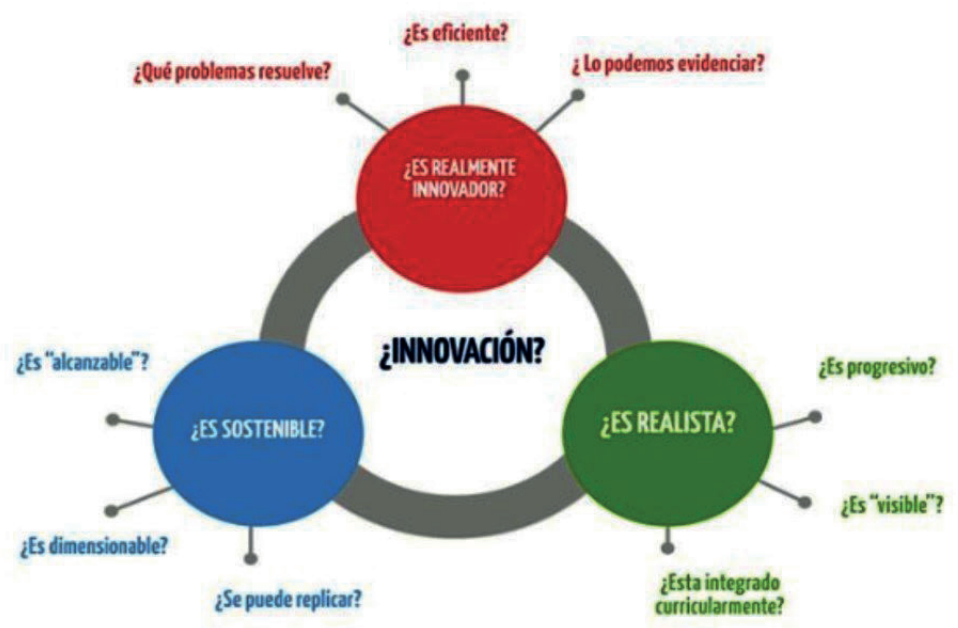

Fuente: Santiago y Bergmann, 2018, p. 18.

En ese orden de ideas, la propuesta de formación binacional fue innovadora, ya que resolvió problemas de formación en competencias digitales que se evidenciaron en el confinamiento producto de las restricciones presentadas desde mayo de 2020, derivadas de los contagios por Covid-19. Fue eficiente, al contemplar las necesidades de formación de la comunidad educativa; necesidades que se convirtieron en insumo fundamental en los cambios que desde 2021 se presentaron en el contexto pedagógico del IYPM. 
Además, es sostenible -segundo interrogante-, en primer lugar, al poder replicarse en cada una de las aulas presenciales y virtuales por la naturaleza de la Clase invertida y al tener apoyo del músculo financiero y administrativo del centro educativo y, por supuesto, alcanzable, la estrategia es una realidad en la institución.

Sobre el tercer y último interrogante para definir el componente innovador, se afirma que es una propuesta realista, se integró al currículo en términos metodológicos, es visible al estar siendo aplicada en las aulas y es progresiva, en la medida que evolucionará y se generarán procesos de aprendizaje simultáneos por pares entre docentes y estudiantes durante los próximos ańos.

En términos particulares, este análisis de resultados, previo a las conclusiones, se enfoca en los elementos de competencia planteados en las fases de diagnóstico y diseńo, cronograma de actividades, material pedagógico virtual compartido, asistencia de estudiantes y criterios aplicados de evaluación.

Con respecto a las competencias, particularmente el EC1, vinculado al saber (ver cuadro 1), en la fase uno del diplomado se abordaron contenidos y se desarrollaron prácticas vinculadas al conocimiento de las variables relacionadas con la metodología Clase invertida, sus términos y conceptos previos como didáctica, lúdica, modelos pedagógicos y otros; se apropiaron los conceptos y se aplicaron progresivamente, a medida que avanzó el curso; las dudas fueron resueltas tanto en sesiones sincrónicas, como en los medios habilitados en el LMS seleccionado.

Es de carácter medular resaltar que las sesiones sincrónicas tuvieron un papel fundamental en el diseńo pedagógico de la propuesta de formación. En las mismas, se compartió el nivel de trabajo autónomo esperado en los tiempos asincrónicos de estudio, se aclararon dudas tanto grupales como particulares, se explicó el tipo de material compartido en plataforma y sus objetivos con respecto a cada elemento de competencia, así como la evolución de las publicaciones en el ambiente virtual de aprendizaje o aula virtual y, por supuesto, el sistema de evaluación. Además, en esas sesiones se generaron diálogos constructivos entre los actores, que fortalecieron la asimilación de la estrategia con aportes desde las distintas realidades.

En esa dirección, en el EC2 -ser-, la Clase invertida fue reconocida por la comunidad educativa como una posibilidad para rea- 
lizar diseños pedagógicos innovadores; lo anterior, desde la segunda fase del diplomado -sesiones 3 a 6-; esta afirmación se consolida con los comentarios en plataforma, diálogos grupales en las sesiones sincrónicas, opiniones de los participantes en la ceremonia virtual de clausura. La implementación de esta megaestrategia en la práctica docente de la universidad comenzó en el primer semestre de 2021.

Finalmente, el EC3 -hacer-; se enfocó en un gran proyecto pedagógico final, que se ligó al diseńo de una estrategia que sintetizara lo aprendido durante el diplomado. De manera concreta, se concluye, luego de evaluar los proyectos presentados y las respectivas sustentaciones, que las estrategias presentadas por los estudiantes se adaptaron a las expectativas, fueron innovadoras y, en su mayoría, adaptaron los principios de la clase invertida, desde los tres elementos de competencia.

El segundo punto de este apartado se liga al cronograma o calendario. El diplomado, tal como se planeó, comenzó con la presentación general en septiembre y finalizó oficialmente el 23 de diciembre del 2020; una semana adicional, debido a que, luego de la semana cuatro no se hizo el encuentro agendado, para que los docentes aprendices pudieran adelantar sus tareas pendientes. Los encuentros sincrónicos se llevaron a cabo los días miércoles de 8:00 a 10:00 p. m., sin contratiempos.

Ahora bien, en los resultados también se aborda el material pedagógico virtual compartido. Todos se construyeron siguiendo los formatos de la institución, así:

- Syllabus o microcurrículo inicial, documento de 15 páginas con información general, competencias, listado de contenidos por semana, modelo pedagógico, procedimientos de estudio, agenda, criterios de evaluación, código de ética y comportamiento, perfil del docente tutor y referencias empleadas.

- Apuntes de unidad: constituidos por recursos virtuales de soporte vinculados a la guía principal; los mismos, junto a las orientaciones del docente, brindaron información relevante de las diferentes unidades que formaron parte de los aprendizajes. 
- Lectura base -guía semanal-: incluyó introducción del curso, organizador gráfico general, calendario e intensidad horaria tanto del trabajo sincrónico como autónomo (ver cuadro 2), elementos de competencia, descripción de actividades de aprendizaje con orientación del tutor para los encuentros sincrónicos, actividades para el trabajo autónomo, requerimientos tecnológicos, perfil del docente tutor y autor de la propuesta. Asimismo, cada unidad presentó su propio organizador gráfico -hechos con la herramienta online Coggle-, introducción de unidad y explicación apoyada por códigos de respuesta rápida $\mathrm{o} Q \mathrm{R}$, que permiten acceder de un modo ágil y sencillo a una determinada información (ComputerHoy, 2014), videos, podcasts, enlaces a textos como libros, artículos científicos, material pedagógico enriquecido, autoevaluación semanal desde formularios en línea, actividades semanales y rúbricas. En la sección final del documento, las referencias y anexos.

- Actividades de aprendizaje orientadas al proyecto final.

- Autoevaluación semanal; con preguntas para identificar progreso individual y grupal. Se prestó apoyo y seguimiento especial a los estudiantes que no mostraban avances en las autoevaluaciones y actividades semanales.

- Grabación semanal de clase, los estudiantes que no podían asistir la revisaban de forma asincrónica en el LMS citado.

Es así como, a través de estos materiales el aprendizaje se hizo notorio, un proceso cognitivo altamente interactivo de construcción personal de significado, donde los alumnos organizaron, filtraron, codificaron, categorizaron y evaluaron la información y construyeron su nuevo conocimiento. Desde dicha perspectiva, el contenido fue percibido como un elemento fundamental que incentivó la construcción de conocimientos y generación de aprendizajes significativos. La autonomía en esa construcción fue fundamental; en consecuencia, la investigación, lectura y codificación de información se consideraron sustanciales en el aprendizaje del estudiante.

La aplicación de los aprendizajes en actividades pedagógicas cotidianas se presentó desde dos perspectivas. En primer lugar, a medi- 
da que avanzó la formación, los docentes participantes aplicaron en la estructuración del proyecto final los conocimientos y habilidades adquiridos; actividad que debía formar parte de su contexto real de trabajo. En consecuencia, fue aplicable desde los contenidos, propósitos de enseñanza, secuencia, metodología -en este caso Clase invertida-, sistema de evaluación y recursos, los seis elementos del currículo propuestos por De Zubiría (2013). En segunda instancia, debían presentar a líderes de programa y directivos de la universidad en el primer semestre de 2021 y, con base en las competencias desarrolladas en el diplomado, propuestas de cambio metodológico en las asignaturas orientadas. La meta, a largo plazo, es realizar procesos de autoevaluación y realimentación constante en los ciclos propedéuticos subsiguientes, para generar mejora continua con aportes de todos los actores involucrados.

Por otra parte, se exponen los criterios de evaluación aplicados. Para ser consistente con la modalidad educativa y las condiciones del curso, la evaluación hizo parte del proceso mismo de aprendizaje. Por tal razón, las actividades y tareas planteadas y desarrolladas por los participantes y su respectiva realimentación fueron evidencia sustancial para el cumplimiento de los objetivos.

El enfoque metodológico de construcción del conocimiento, busca "que el ser humano consiga integrar el saber conocer, saber hacer, saber ser y saber convivir" (Moreno, 2012, p. 252). De ahí que se evaluara la participación tanto en los foros como en las actividades que reflejaron mayormente que dicho proceso de construcción se presentara realmente. Cada actividad de aprendizaje contó con una rúbrica detallada con categorías/aspectos a evaluar que cada participante debía revisar para identificar sus objetivos. El sistema de evaluación, aprobación y titulación fue acorde al reglamento interno de la universidad. El cuadro 3 sintetiza los criterios generales.

Cuadro 3. Criterios generales de evaluación

\begin{tabular}{|l|c|l|}
\hline \multicolumn{1}{|c|}{ ASPECTO POR EVALUAR } & PORCENTAJE & \multicolumn{1}{|c|}{ DESCRIPCIÓN } \\
\hline Asistencia a encuentros sincrónicos & 40 & $\begin{array}{l}\text { Presentación de diplomado, sesiones sincrónicas, } \\
\text { y sustentaciones finales. }\end{array}$ \\
\hline Actividades grupales de formación & 40 & Proyecto pedagógico final, con base en rúbrica \\
\hline Foros y actividades semanales & 20 & $\begin{array}{l}\text { Diligenciamiento de autoevaluaciones y } \\
\text { ejercicios propuestos cada semana. }\end{array}$ \\
\hline
\end{tabular}

Fuente: elaboración propia. 
Concretamente, sobre la matrícula y asistencia, se registraron 39 estudiantes en listas; cuatro no cumplieron con los requisitos mínimos establecidos desde la fase inicial, dos con cero asistencias a encuentros sincrónicos y dos con una. Luego del seguimiento, se comprobó que las causas se asociaron a dificultades de índole familiar, salud y sobrecarga laboral. Con respecto a los demás, 15 estudiantes asistieron a la totalidad de los encuentros, 15 a diez encuentros $(83.3 \%)$, dos a ocho encuentros $(66,6 \%)$ y tres estudiantes a la mitad; con ellos se hizo un trabajo paralelo de seguimiento y actualización para que finalizaran el curso sin contratiempos. En otras palabras, 30 de 35 estudiantes estuvieron en la gran mayoría o todas las sesiones sincrónicas.

Para finalizar, se resalta que, de los 39 estudiantes iniciales, sólo 35 fueron tenidos en cuenta para la estadística final, debido a que cuatro de ellos abandonaron en la fase inicial -descritos en el punto anterior-. Sobre este grupo, la aprobación final fue de 82.9\% (29 estudiantes) y la reprobación de $17.1 \%$ (seis estudiantes); las causas de los incumplimientos ligados a la reprobación, luego del seguimiento en enero de 2021, se resumieron a falta de tiempo por situaciones laborales (cuatro estudiantes) y bajo nivel de interés por el tema del diplomado (dos estudiantes).

Luego de analizar los proyectos finales y aplicar la prueba de salida, se evidenció que el principal desarrollo de competencias se presentó en los primeros tres aspectos medulares de formación: planificación y ejecución de enseñanza híbrida con TIC; selección y creación de objetos virtuales de aprendizaje, y diseńo y desarrollo de entornos digitales de enseñanza. Con respecto al cuarto elemento -tutoría y evaluación en línea-, se compartieron conceptos, herramientas, aplicaciones y estrategias para la mediación con tecnología sobre las dos acciones; sin embargo, al no aplicarse aún los proyectos planteados en la realidad pedagógica de los docentes aprendices y sus estudiantes, en la sección final del diplomado no se pudo comprobar la eficiencia de las estrategias de evaluación y tutoría descritas en los mismos, a pesar de haber demostrado en el planteamiento la apropiación de conceptos. 


\section{CONCLUSIONES}

La necesidad de formación en Competencias Digitales Docentes cubierta por el diplomado Clase invertida, nuevas tendencias en educación mediadas por tecnología, se hizo más notoria al enfrentar los efectos colaterales de la Covid-19 en las prácticas pedagógicas del IYPM; por esa razón, la formación se enfocó en cuatro aspectos medulares: planificación y ejecución de enseñanza híbrida con TIC; selección y creación de objetos virtuales de aprendizaje; diseño y desarrollo de entornos digitales de enseñanza, y posibilidades de tutoría y evaluación en línea.

La evaluación diagnóstica se sustentó en dos líneas de acción. En primer lugar, la caracterización de la población e ideas fuerza, para identificar el tipo de percepción frente a algunos conceptos y niveles de conocimiento, habilidades y destrezas con respecto a los mismos, y desarrollo de competencia digital docente. La segunda línea de acción del diagnóstico fue el diálogo no estructurado con directivos y personal administrativo de la universidad, durante los meses de planificación y diseño de la propuesta. Lo anterior, con el fin de identificar necesidades particulares de aprendizaje y competencias previas al diplomado.

La experiencia de formación binacional generó un balance positivo, concretamente, en la acogida, continuidad e impacto del ciclo académico expuesto en este artículo. Acogida por la comunidad mexicana que hizo parte del público objetivo específico; el 82.9\% de aprobación y titulación así lo ratifica. Continuidad, por la aprobación de la siguiente cohorte de formación para el segundo semestre de 2021, e impacto, porque es una realidad el cambio pedagógico en los escenarios académicos del IYPM con base en la metodología Clase invertida.

El diseño curricular se enfocó en seis elementos -contenidos, secuencia, metodología, recursos, evaluación y propósitos de aprendizaje- que estructuraron las tres fases de la propuesta de formación -diseño, ejecución y análisis-síntesis-; todo tuvo una duración de diez meses. La relevancia del acompañamiento, apoyo, gestión, publicación y promoción del diplomado por parte de los directivos y personal administrativo del IYPM fue de vital importancia en la experiencia de formación. 
La adaptación cultural se convirtió en uno de los principales retos en esta propuesta de formación dirigida desde Colombia. El análisis diagnóstico inicial de cultura institucional, dinámicas organizacionales de comunicación - plataforma, formatos, tiempos sincrónicos y autónomos de trabajo-, características generales y particulares de los docentes aprendices y por supuesto, necesidad de formación en competencias digitales, fue el cimiento tanto de la estructura planteada como de la didáctica propuesta en los espacios grupal e individual y elaboración del material pedagógico compartido en distintos formatos.

Con respecto a la competencia general y sus tres elementos, se abordaron contenidos y se desarrollaron prácticas vinculadas al conocimiento de las variables relacionadas con la metodología Clase invertida, sus términos y conceptos previos (saber); se apropiaron los conceptos y se aplicaron progresivamente, a medida que avanzó el curso. La Clase invertida fue reconocida por la comunidad educativa como una posibilidad para realizar diseños pedagógicos innovadores (ser); esta afirmación se consolida con la implementación de esta megaestrategia en la práctica docente de la universidad a partir del primer semestre de 2021. Los proyectos presentados por los docentes aprendices como producto final (hacer), cumplieron con las expectativas planteadas inicialmente; fueron innovadores y en su mayoría adaptaron los principios de la clase invertida. El cambio de perspectiva con respecto a sus prácticas tradicionales fue evidente.

A través del material pedagógico virtual compartido se evidenció un proceso cognitivo altamente interactivo de construcción personal de significado, donde los alumnos organizaron, filtraron, codificaron, categorizaron y evaluaron la información y construyeron su nuevo conocimiento. Desde dicha perspectiva, el contenido fue percibido como un elemento fundamental que incentivó la construcción de conocimientos y generación de aprendizajes significativos.

La autonomía en el proceso de construcción del conocimiento fue fundamental; en consecuencia, la investigación, lectura y codificación de la información se consideraron aspectos sustanciales en el aprendizaje del estudiante.

Se comparte esta propuesta con el fin de que se convierta en revulsivo de nuevas posibilidades de formación en competencias digitales de las dispares comunidades académicas de educación básica 
y superior en Latinoamérica, desde la metodología Clase invertida, aula inversa o Flipped Classroom; claro está, lo anterior, desde las características particulares en términos de recursos, ubicación geográfica, nivel de cualificación, saberes previos y expectativas de los actores involucrados.

Finalmente, el principal desarrollo de competencias se presentó en los primeros tres aspectos medulares de formación: planificación y ejecución de enseñanza híbrida con TIC, selección y creación de objetos virtuales de aprendizaje, y diseño y desarrollo de entornos digitales de enseńanza. Con respecto al cuarto elemento -tutoría y evaluación en línea-, se compartieron conceptos, herramientas, aplicaciones y estrategias para la mediación con tecnología sobre las dos acciones; sin embargo, a pesar de haber demostrado en el planteamiento la apropiación de conceptos, al no aplicarse aún los proyectos planteados en la realidad pedagógica de los docentes aprendices y sus estudiantes, no se pudo comprobar la eficiencia de las estrategias de evaluación y tutoría descritas en los mismos.

\section{REFERENCIAS}

Area-Moreira, M. (2019). Competencia digital docente. Canal ULLaudiovisual-Universidad de La Laguna. https://youtu.be/vpNzBuIbBMw Banoy, W. (2020). Clase Invertida, nuevas tendencias en educación mediadas por tecnología. Bogotá: Corporación Universitaria minuto de Dios, Uniminuto.

Banoy, W. (2021). Píldora 7: videos interactivos con VIZIA. Canal TEAM_4.0, Tecnología, Enseñanza, Aprendizaje y Motivación. https:// youtu.be/knub0rkoUn0

Blázquez-Merino, M., y Tarazaga, J. (2017). La estructura de prácticas basada en la taxonomía de Bloom-Anderson. Portal UNED. https:// canal.uned.es/video/5a6f468eb1111f010a8b4575

Cabero, J., y Aguaded, J. (2013). Tecnologías y medios para la educación en la e-sociedad. Madrid: Alianza Editorial.

Cabero, J., y Martínez Gimeno, A. (2019). Las Tecnologías de la Información y Comunicación y la formación inicial de los docentes. Modelos y competencias digitales. Profesorado. Revista de Curriculum y Formación de Profesorado, 23(3), 247-268. https://10.30827/profesorado. v23i3.9421 
ComputerHoy (2014). ¿Qué son los códigos QR y cómo funcionan? https:// computerhoy.com/noticias/internet/que-son-codigos-qr-como-funcionan-14973

Conecta-TE (2013). Algunos apuntes sobre la noción de "Aula Invertida" o "The Flipped Classroom". Bogotá, Colombia: Centro de Innovación en Tecnología y Educación. Universidad de los Andes. https://conectate. uniandes.edu.co/index.php/innovacion/tendencias/clase-invertida

Corona, A., Altamirano, M., López Ortega, M., y González González, O. (2019). Analítica del aprendizaje y las neurociencias educativas: nuevos retos en la integración tecnológica. Revista Iberoamericana de Educación, 80(1), 31-54. https://doi.org/10.35362/rie8013428

De Zubiría, J. (2006). Los modelos pedagógicos, hacia una pedagogía dialogante. Bogotá: Editorial Magisterio.

De Zubiría, J. (2015, septiembre). Reseña, modelos pedagógicos. Canal Magisterio TV. https://www.youtube.com/watch?v=5pS8XrTuz8E

De Zubiría, J. (2013). ¿Cómo diseñar un currículo por competencias? Bogotá: Editorial Magisterio.

Flipped Learning Network (FLN) (2014). The Four Pillars of F-L-I-P. https://flippedlearning.org/wp-content/uploads/2016/07/FLIP_handout_FNL_Web.pdf

García-Barrera, A. (2013). El aula inversa: cambiando la respuesta a las necesidades de los estudiantes. Revista Avances en Supervisión Educativa, (19), 1-8. https://doi.org/10.23824/ase.v0i19.118

Kraus, G., Formichella, M., y Alderete, M. (2019). El uso del Google Classroom como complemento de la capacitación presencial a docentes de nivel primario. Revista Iberoamericana de Tecnología en Educación y Educación en Tecnología, 24(1), 79-90. https://doi. org/10.24215/18509959.24.e09

Moreno, C. (2012). La construcción del conocimiento: un nuevo enfoque de la educación actual. Sophia, Colección de Filosofía de la Educación, (13), 251-267. https://www.redalyc.org/articulo. oa?id=441846102011

Morin, E. (2011). Introducción al pensamiento complejo. París: Editorial ESPF.

Morin, E., y Delgado, C. (2017). Reinventar la educación. Abrir caminos a la metamorfosis de la humanidad. Hermosillo, México: Desde Abajo.

Orduz, R. (2021, 29 de marzo). La brecha digital y la pandemia: mayor inequidad. El Espectador. https://www.elespectador.com/opinion/labrecha-digital-y-la-pandemia-mayor-inequidad/ 
Ríos, D., y Herrera, D. (2017). Los desafíos de la evaluación por competencias en el ámbito educativo. Revista Educação e Pesquisa, 43(4), 1073-1086. http://dx.doi.org/10.1590/S1678-4634201706164230

Rodríguez, M. (2017). Currículum, educación y cultura en la formación docente del siglo XXI desde la complejidad. Revista Educación y Humanismo, 19(33), 425-440. http://dx.doi.org/10.17081/eduhum.19.33.2654

Robinson, K., y Aronica, L. (2015). Escuelas creativas, la revolución que está transformando la educación. Barcelona: Grijalbo.

Ruz, I. (2018). Evaluación para el aprendizaje. Revista Educación Las Américas, 6(2018), 13-28. https://revistas.udla.cl/index.php/rea/article/ view/22

Santiago, R., Díez, A., y Andía, L. (2017). Flipped Classroom, 33 experiencias que ponen patas arriba el aprendizaje. Barcelona: Oberta UOC Publishing SL.

Santiago R., y Bergmann, J. (2018). Aprender al revés, flipped learning 3.0 y metodologías activas en el aula. Barcelona: Paidós Educación.

Solórzano, Y. (2017). Aprendizaje autónomo y competencias. Revista científica Dominio de las Ciencias, 3(Extra.), 241-253. https://dialnet.unirioja.es/servlet/articulo?codigo $=5907382$

Torras, M. (2018). Las plataformas LMS; definición, características, tipos y plataformas más utilizadas. Valencia, España: Universidad Internacional de Valencia. https://recursos.universidadviu.es/guia-plataformas$\operatorname{lms}$

Valero, R., Palacios-Rozo, J., y González-Silva, R. (2019). Tecnologías de la Información y la Comunicación y los Objetos Virtuales de Aprendizaje: un apoyo a la presencialidad. Revista Vinculos, 16(1). https://doi. org/10.14483/2322939X.15537

Zabalza, M. (2007). Competencias docentes del profesorado universitario. Calidad y desarrollo profesional. Madrid: Narcea. 